\title{
Semi-orthogonal wavelets that behave like fractional differentiators
}

\author{
Dimitri Van De Ville ${ }^{1}$, Thierry Blu${ }^{1}$, Brigitte Forster ${ }^{2}$, Michael Unser ${ }^{1}$ \\ ${ }^{1}$ Biomedical Imaging Group, École Polytechnique Fédérale de Lausanne, Switzerland \\ ${ }^{2}$ Centre for Mathematical Sciences, Munich University of Technology, Germany
}

\begin{abstract}
The approximate behavior of wavelets as differential operators is often considered as one of their most fundamental properties. In this paper, we investigate how we can further improve on the wavelet's behavior as differentiator. In particular, we propose semi-orthogonal differential wavelets. The semi-orthogonality condition ensures that wavelet spaces are mutually orthogonal. The operator, hidden within the wavelet, can be chosen as a generalized differential operator $\partial_{\tau}^{\gamma}$, for a $\gamma$-th order derivative with shift $\tau$. Both order of derivation and shift can be chosen fractional. Our design leads us naturally to select the fractional B-splines as scaling functions. By putting the differential wavelet in the perspective of a derivative of a smoothing function, we find that signal singularities are compactly characterized by at most two local extrema of the wavelet coefficients in each subband. This property could be beneficial for signal analysis using wavelet bases. We show that this wavelet transform can be efficiently implemented using FFTs.
\end{abstract}

Keywords: Wavelet Bases, Generalized Fractional Splines, Differential Operators

\section{INTRODUCTION}

From the very beginning of wavelets, researchers have recognized the strong connection between wavelets and differential operators. ${ }^{1-3}$ Indeed, the differentiation behavior of the wavelet is essential for two highly praised properties of the wavelet transform; i.e., locality and sparsity. Consequently, transient features such as discontinuities are characterized by wavelet coefficients in their neighborhood only, the same way as a derivative acts locally too. In coding applications, the sparse representations that are obtained can be exploited successfully to compactly represent the information content. In signal and image analysis, the differentiation property of wavelets can also be exploited explicitly. Most notably, Mallat et al. ${ }^{4-6}$ used wavelets to reconstruct a signal from its wavelet coefficients' zero-crossings (when the wavelet acts like a second-order derivative) or their modulus maxima (when it acts like a first-order derivative). Due to the lack of shift-invariance of the wavelet transform, Mallat and Zhong ${ }^{5}$ had to propose a redundant and nonorthogonal version, thus extending wavelet bases to wavelet frames. While the redundant wavelet transform makes the analysis of the signal's wavelet coefficients easier, it has some obvious shortcomings: over-completeness and a non-unique inverse transform.

In this paper, we are interesting in better controlling the differentiation behavior of the wavelet. For that purpose, we propose a new type of semi-orthogonal wavelet. The semi-orthogonality condition forces the wavelet spaces to be orthogonal to one another. As for the scaling function, we naturally pick generalized fractional Bsplines, ${ }^{7,8}$ which are intimately related to the broad class of differential operators $\partial_{\tau}^{\gamma}$, for a $\gamma$-th order derivative with shift $\tau$. Putting the wavelet in the perspective of a derivative of a smoothing function, we show that the differential wavelet is related to the cardinal interpolating spline wavelet. We also comment on the fact that a signal singularity gives rise to at most two local extrema per scale in the wavelet domain, a feature that opens up new ways to analyze a signal using wavelet bases. Finally, we briefly describe the implementation of our wavelet transform and present an illustrative example.

Send correspondence to Dimitri Van De Ville, E-mail: Dimitri.VanDeVille@epfl.ch, Telephone: +41 216935142 


\section{SEMI-ORTHOGONAL B-SPLINE WAVELETS}

\subsection{Fractional B-splines}

We consider the generalized fractional B-spline of degree $\alpha \geq 0(\alpha \in \mathbb{R})$, and shift $\tau \in \mathbb{R}$, which are most conviently defined in the Fourier domain:

$$
\hat{\beta}_{\tau}^{\alpha}(\omega)=\left(\frac{1-e^{j \omega}}{-j \omega}\right)^{\frac{\alpha+1}{2}-\tau}\left(\frac{1-e^{-j \omega}}{j \omega}\right)^{\frac{\alpha+1}{2}+\tau} .
$$

The associated approximation space $\mathcal{V}_{i}$ at a scale $i$ is

$$
\mathcal{V}_{i}=\left\{s(x)=\sum_{k} c[k] \beta_{\tau}^{\alpha}\left(\frac{x}{2^{i}}-k\right) ; c[k] \in l_{2}(\mathbb{Z})\right\} .
$$

The above fractional B-splines are closely related to fractional differential operators. We define such a generalized operator in the sense of distributions as a multiplication in the Fourier domain:

$$
\partial_{\tau}^{\gamma} f(x)=\int_{-\infty}^{\infty} \underbrace{(-j \omega)^{\frac{\gamma}{2}-\tau}(j \omega)^{\frac{\gamma}{2}+\tau}}_{=: \hat{\delta}_{\tau}^{\gamma}(\omega)} \hat{f}(\omega) e^{j \omega x} \frac{\mathrm{d} \omega}{2 \pi} .
$$

We notice that the complex conjugate of $\hat{\delta}_{\tau}^{\gamma}(\omega)$ equals $\hat{\delta}_{\tau}^{\gamma}(-\omega)$. When we let this operator act on a B-spline, we get

$$
\partial_{\tau}^{\gamma} \beta_{\tau}^{\alpha}=\Delta_{\tau}^{\gamma} \beta_{\tau}^{\alpha-\gamma}
$$

where $\Delta_{\tau}^{\gamma}$ is the generalized fractional finite difference operator whose transfer function is

$$
\hat{\Delta}_{\tau}^{\gamma}(z)=(1-z)^{\frac{\gamma}{2}-\tau}\left(1-z^{-1}\right)^{\frac{\gamma}{2}+\tau},
$$

with $z=e^{j \omega}$. While it is usual to index the splines by their degree, we will more often refer to their order of approximation, which is $\gamma=\alpha+1$, and which expresses a more fundamental property of B-splines. ${ }^{9,10}$

The fractional B-splines satisfy the three admissibility conditions for a scaling function ${ }^{7,8}$ :

(i) They generate a Riesz basis of $V_{0}$; i.e., the autocorrelation filter

$$
A\left(e^{j \omega}\right)=\sum_{k}\left|\hat{\beta}_{\tau}^{\alpha}(\omega+2 \pi k)\right|^{2}
$$

is bounded by two constants $0<C_{0} \leq\left|A\left(e^{j \omega}\right)\right| \leq C_{1}<\infty$.

(ii) They fulfill the partition of unity:

$$
\sum_{n} \beta_{\tau}^{\alpha}(x-n)=1
$$

(iii) They satisfy a two-scale relation; i.e., there exists a scaling filter that relates the B-splines at two consecutive scales:

$$
\beta_{\tau}^{\alpha}\left(\frac{x}{2}\right)=\sum_{k} h[k] \beta_{\tau}^{\alpha}(x-k) .
$$

These equations are valid pointwise for $\alpha>0$ and almost everywhere for $\alpha=0$. It is not difficult to see that the $z$-transform of the filter $h[k]$ is given by

$$
H(z)=2^{-\alpha} \frac{\hat{\Delta}_{\tau}^{\alpha+1}\left(z^{2}\right)}{\hat{\Delta}_{\tau}^{\alpha+1}(z)}=2^{-\alpha}(1+z)^{\frac{\alpha+1}{2}-\tau}\left(1+z^{-1}\right)^{\frac{\alpha+1}{2}+\tau} .
$$




\subsection{Fractional B-spline wavelets}

The design strategy for the semi-orthogonal spline wavelet transform, which was initially proposed by Unser $e t$ $a l .,{ }^{11}$ consists in selecting the wavelet space

$$
\mathcal{W}_{i}=\operatorname{span}_{n \in \mathbb{Z}}\left\{\psi\left(\frac{x}{2^{i}}-n\right)\right\}
$$

as the orthogonal complement of $\mathcal{V}_{i}$ in $\mathcal{V}_{i-1}$, which are both generated by the non-orthonormalized B-splines. Using the fact that the wavelet $\psi$ at scale $i=1$ is part of $\mathcal{V}_{0}$, i.e.,

$$
\psi\left(\frac{x}{2}\right)=\sum_{k} w[k] \beta_{\tau}^{\alpha}(x-k)
$$

and expressing the orthogonality between $\mathcal{V}_{i}$ and $\mathcal{W}_{i}$, results into

$$
W(z) H\left(z^{-1}\right) A(z)+W(-z) H\left(-z^{-1}\right) A(-z)=0 .
$$

which requires $W(z) H\left(z^{-1}\right) A(z)$ to be an odd polynomial.

Proposition 1. For any given generalized B-spline $\beta_{\tau}^{\alpha}$, one can specify a corresponding semi-orthogonal spline wavelet $\psi$, parametrized by some filter $Q(z)$, that has the following properties:

(i) Its Fourier domain expression is given by

$$
\hat{\psi}(2 \omega)=W\left(e^{j \omega}\right) \frac{\hat{\beta}_{\tau}^{\alpha}(\omega)}{2},
$$

with $W(z)=z^{-1} Q\left(z^{2}\right) H\left(-z^{-1}\right) A(-z)$.

(ii) It generates a Riesz basis whenever $Q\left(e^{j \omega}\right)$ is bounded by $C_{0} \leq\left|Q\left(e^{j \omega}\right)\right| \leq C_{1}$ for some constants $0<$ $C_{0}, C_{1}<\infty$.

(iii) For lower frequencies, it follows the behavior of the differential operator that is related to the B-spline:

$$
\hat{\psi}(\omega)=C_{2} \cdot \hat{\delta}_{\tau}^{\gamma}(-\omega)+O\left(|\omega|^{\gamma+1}\right), \quad \text { as } \omega \rightarrow 0 .
$$

The proof will be given in a future paper. ${ }^{12}$ This proposition implies that the semi-orthogonal wavelet always behaves asymptotically as a fractional differential operator. Consequently, analyzing a signal $f(x)$ with this wavelet yields samples of the operator $\partial_{\tau}^{\gamma}$ applied to a smoothed version of the input signal:

$$
\langle f(x), \psi(x-k)\rangle=\partial_{\tau}^{\gamma}\{\phi * f\}(k)
$$

where the smoothing function is defined in the Fourier domain as $\hat{\phi}(\omega)=\hat{\psi}(\omega) / \hat{\delta}_{\tau}^{\gamma}(-\omega)$.

When $\alpha$ is an integer, and $\tau=(\alpha+1) / 2$, one obtains the traditional "B-spline wavelet" $\operatorname{setting} Q(z)=1$, which corresponds to the wavelet filter $W(z)$ with the shortest possible support ${ }^{11}$ :

$$
W(z)=z^{-1} H\left(-z^{-1}\right) A(-z) .
$$

These wavelets are also defined for other values of $\alpha$ and $\tau$, buth the finite-support argument is no longer always valid; i.e., the corresponding filters $H(z)$ and $A(z)$ have generally infinite support. Therefore, we are looking for an alternative way of exploiting the degree of freedom of $Q\left(z^{2}\right)$ in the design. 


\section{IMPROVED OPERATOR BEHAVIOR}

\subsection{Differential wavelets}

We now show that the asymptotical behavior of the B-spline as differential operator can be significantly improved for a suitable choice of $Q\left(z^{2}\right)$.

Proposition 2. Given a generalized B-spline $\beta_{\tau}^{\alpha}$ as scaling function, we define the corresponding semiorthogonal differential wavelet $\psi$ by choosing

$$
Q\left(z^{2}\right)=\frac{2^{2 \gamma}}{A(z) A(-z)}
$$

Then the wavelet is characterized in the Fourier domain as

$$
\hat{\psi}(\omega)=\hat{\delta}_{\tau}^{\gamma}(-\omega) \cdot \frac{e^{-j \omega}}{1+|\omega|^{2 \gamma} \sum_{k \neq 0} \frac{1}{|\omega+4 \pi k|^{2 \gamma}}},
$$

which is independent of the localization operator of the scaling function and closely matches the differential operator over a larger range of $\omega$ than usual. In particular, we have

$$
\hat{\psi}(\omega)=\hat{\delta}_{\tau}^{\gamma}(-\omega) \cdot e^{-j \omega}+O\left(|\omega|^{3 \gamma}\right), \quad \text { as } \omega \rightarrow 0 .
$$

Again, the proof will be given elsewhere. ${ }^{12}$ When comparing the B-spline with the differential wavelet, we see that the order of the second term of the Taylor development at $\omega=0$ has increased from $\gamma+1$ to $3 \gamma$. In Fig. 1 (a), we show the frequency response of both wavelets together with that of the corresponding differential operator. First, we note that the B-spline and differential wavelets coincide for $\gamma=1$. For $\gamma>1$, the differential wavelet matches the operator behavior more closely than the B-spline wavelet does. The example in Fig. 1 (b) illustrates how the differential wavelet coincide with the pure differential operator in the Fourier domain over a large range of $\omega$. These plots hold for every shift $\tau$. It is also interesting to look at the wavelet shape in the spatial domain. In Fig. 2 (a), the symmetric wavelets for $\gamma=2$ (i.e., the "linear" wavelets) are shown. The differential wavelet has a theoretical infinite support but stays in practice very similar to the B-spline wavelet. For higher degrees, such as $\gamma=3.5$ (Fig. 2 (b)), the difference between both wavelets increases. Notice that the central frequency of the differential wavelet is higher.

We can further refine the choice of $Q\left(z^{2}\right)$ to approximate the operator even better. We propose the following viable (but non-unique) procedure.

Proposition 3. Given a generalized B-spline $\beta_{\tau}^{\alpha}$ as scaling function, then the improved differential wavelet $\psi$ corresponding to the particular choice

$$
Q\left(z^{2}\right)=\frac{2^{2 \gamma}}{A(z) A(-z)} \cdot \underbrace{\frac{B_{2 \gamma}(z) B_{\gamma^{\prime}}(z)+B_{2 \gamma}(-z) B_{\gamma^{\prime}}(-z)}{B_{2 \gamma+\gamma^{\prime}}(z)+B_{2 \gamma+\gamma^{\prime}}(-z)}}_{=: R_{\gamma^{\prime}}\left(z^{2}\right)},
$$

with

$$
B_{\gamma^{\prime}}\left(e^{j \omega}\right)=\sum_{k} \frac{1}{|\omega+2 \pi k|^{\gamma^{\prime}}},
$$

where $\gamma^{\prime} \geq 2 \gamma$, matches the operator in the Fourier domain as

$$
\hat{\psi}(\omega)=\hat{\delta}_{\tau}^{\gamma}(-\omega) \cdot e^{-j \omega}+O\left(|\omega|^{\gamma+\gamma^{\prime}}\right), \quad \text { as } \omega \rightarrow 0 .
$$

As can be seen from Fig. 3, the differentiation behavior is improved but the gain quickly becomes marginal as the order $\gamma$ increases. Therefore, we will focus now on the basic differential wavelet, as introduced before. 
(a) $\gamma=1$

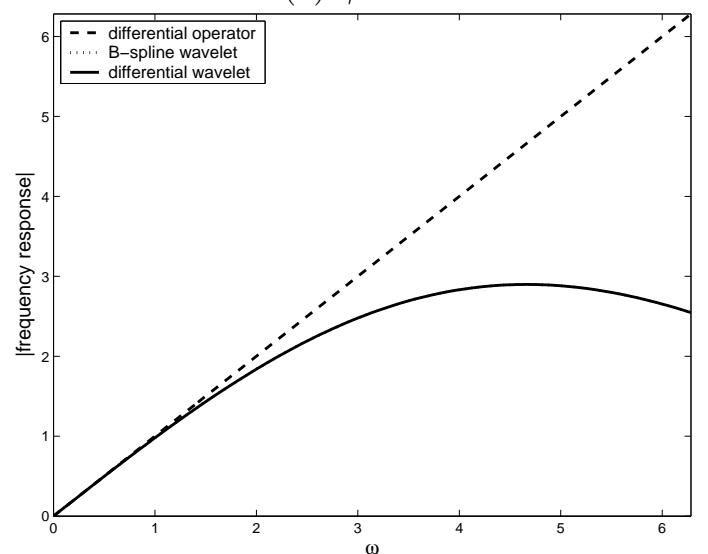

(b) $\gamma=3.5$

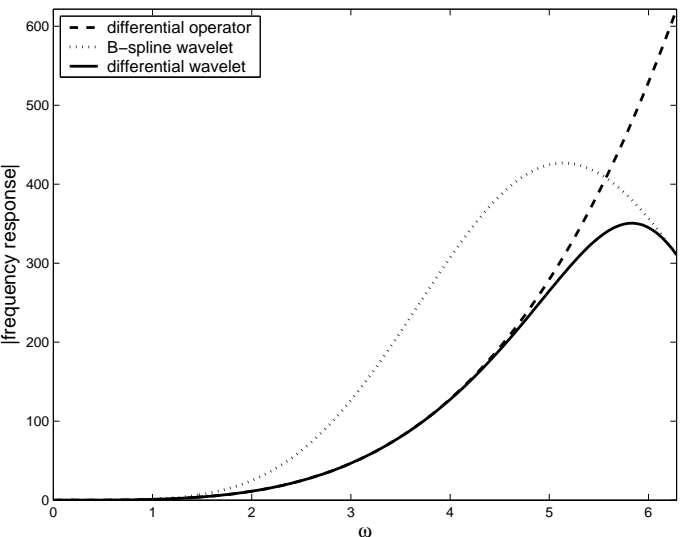

Figure 1. Modulus of the frequency response of the B-spline wavelet and differential wavelet versus the differential operator.

(a) $\gamma=2$

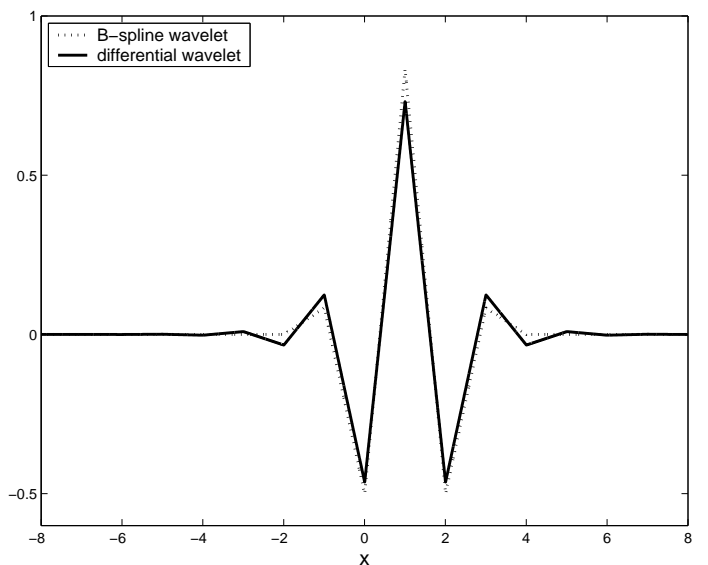

(b) $\gamma=3.5$

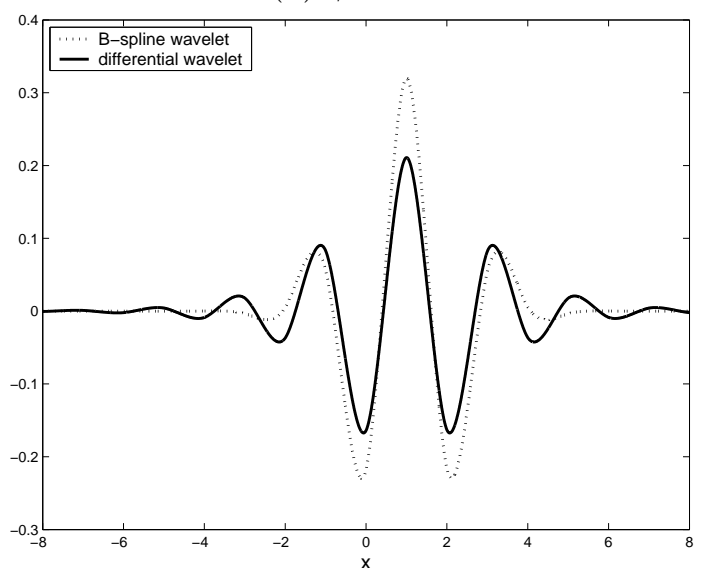

Figure 2. The B-spline and differential wavelet in the spatial domain. Notice how the differential wavelet stays essentially localized.

(a) $\gamma=1$

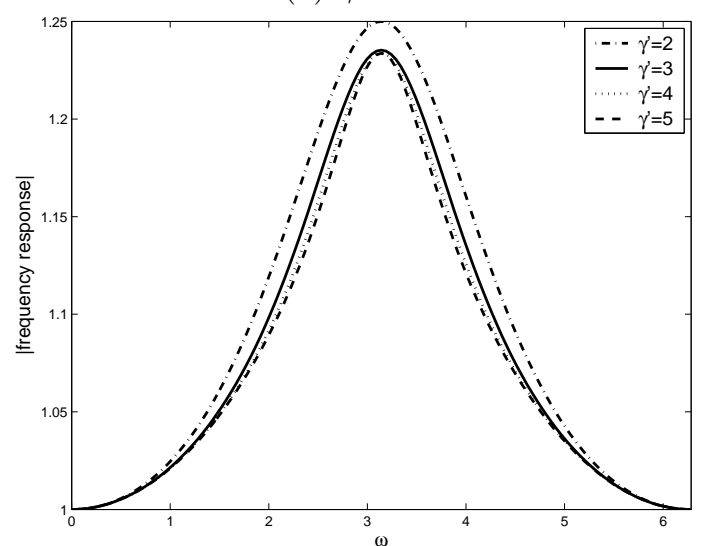

(b) $\gamma=2$

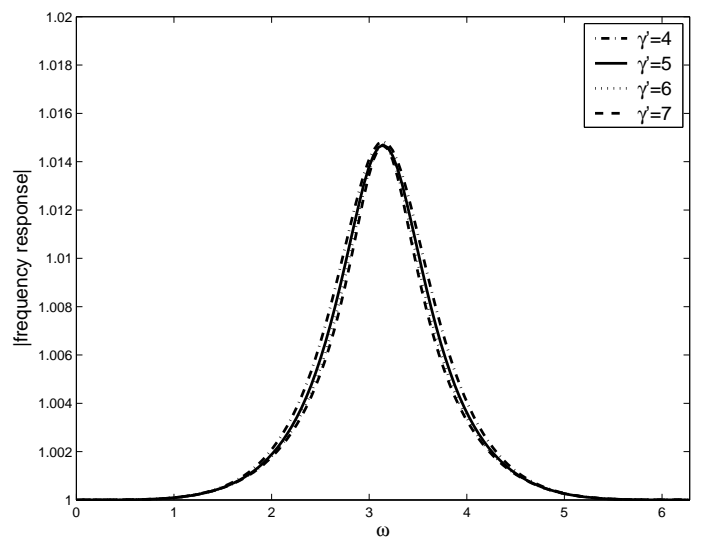

Figure 3. Frequency response of the improvement filter $R_{\gamma^{\prime}}\left(e^{j \omega}\right)$ for (a) $\gamma=1$, (b) $\gamma=2$. 


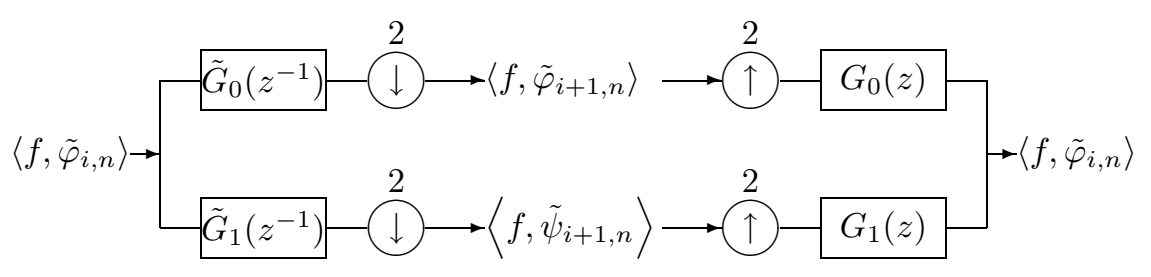

Figure 4. The discrete wavelet transform for one iteration.

\subsection{Properties}

It is instructive to consider the differential wavelet from the perspective of a "derivative of a smoothing function". Given the choice for $Q\left(z^{2}\right)$, we rewrite the differential wavelet in the Fourier domain as

$$
\hat{\psi}(2 \omega)=\hat{\delta}_{\tau}^{\gamma}(-\omega) \cdot 2^{\gamma} e^{-j \omega} \frac{\hat{\beta}_{0}^{2 \gamma-1}(\omega)}{A\left(e^{j \omega}\right)} .
$$

where $1 / A\left(e^{j \omega}\right)$ is the interpolation prefilter of the $2 \gamma$-th order symmetric B-spline. Therefore, we obtain the following form of the differential wavelet:

$$
\psi(x)=\partial_{\tau}^{\gamma} \beta_{\mathrm{int}}^{2 \gamma-1}(2 x-1) .
$$

We recognize the generalized differential operator that comes automatically with the choice of the scaling function. We also see that the smoothing function is the interpolating B-spline of twice the order $\gamma$. In fact, the differential wavelets coincide with the cardinal spline wavelets that were proposed by Chui and Wang ${ }^{13}$ for causal B-spline of integer order. Here, we have obtained an extension to generalized differential operators (fractional orders and shifts) and a method to improve the operator-like behavior even further.

Since the interpolating B-spline rapidly converges to the sinc-function, ${ }^{14}$ samples of it at positions $k \in \mathbb{Z}$ will all the same sign, while samples of its derivatives could be monomodal or bimodal only. Therefore, the wavelet decomposition of a singularity could turn out to have a particularly simple characterization. This property yields an interesting theoretical link with soliton theory and non-linear differential equations. ${ }^{12}$

\section{IMPLEMENTATION}

In practice, the wavelet decomposition algorithm is translated into an efficient filterbank implementation using the scaling and wavelet filters. Figure 4 shows one iteration of the wavelet decomposition. To have the operatorlike behavior on the input signal, one should use the differential wavelet at the analysis side $\tilde{\psi}$, and its dual at the synthesis side. In that case, the filters of Fig. 4 are

$$
\begin{array}{llrl}
\tilde{G}_{0}(z)=H(z), & & G_{0}(z)=H(z) \frac{A(z)}{A\left(z^{2}\right)}, \\
\tilde{G}_{1}(z)=2^{2 \gamma} z^{-1} \frac{H\left(-z^{-1}\right)}{A(z)}, & & G_{1}(z)=2^{4 \gamma-6} z^{-1} H\left(-z^{-1}\right) \frac{A(z) A\left(-z^{-1}\right)}{A\left(z^{2}\right)} .
\end{array}
$$

We use a fast FFT-based algorithm to efficiently implement these filters with periodic boundary conditions. ${ }^{15}$

In Fig. 5, we show an example for a piecewise polynomial signal. Although this is a non-redundant wavelet transform with high order $(\gamma=5)$, we note that the singularities of the signal are characterized by one or two extrema only in each subband.

\section{CONCLUSION}

In this paper, we have introduced a new type of wavelets, which allows us to precisely tune the $\gamma$-th order differentiation behavior of the wavelet. Due to the semi-orthogonality condition, the wavelet spaces are orthogonal to one another, which is a useful property for many applications. The differential wavelet, including its extension to multiple dimensions using polyharmonic B-spline ${ }^{16}{ }^{16}$ will be studied in more detail in a future paper. ${ }^{12}$ 


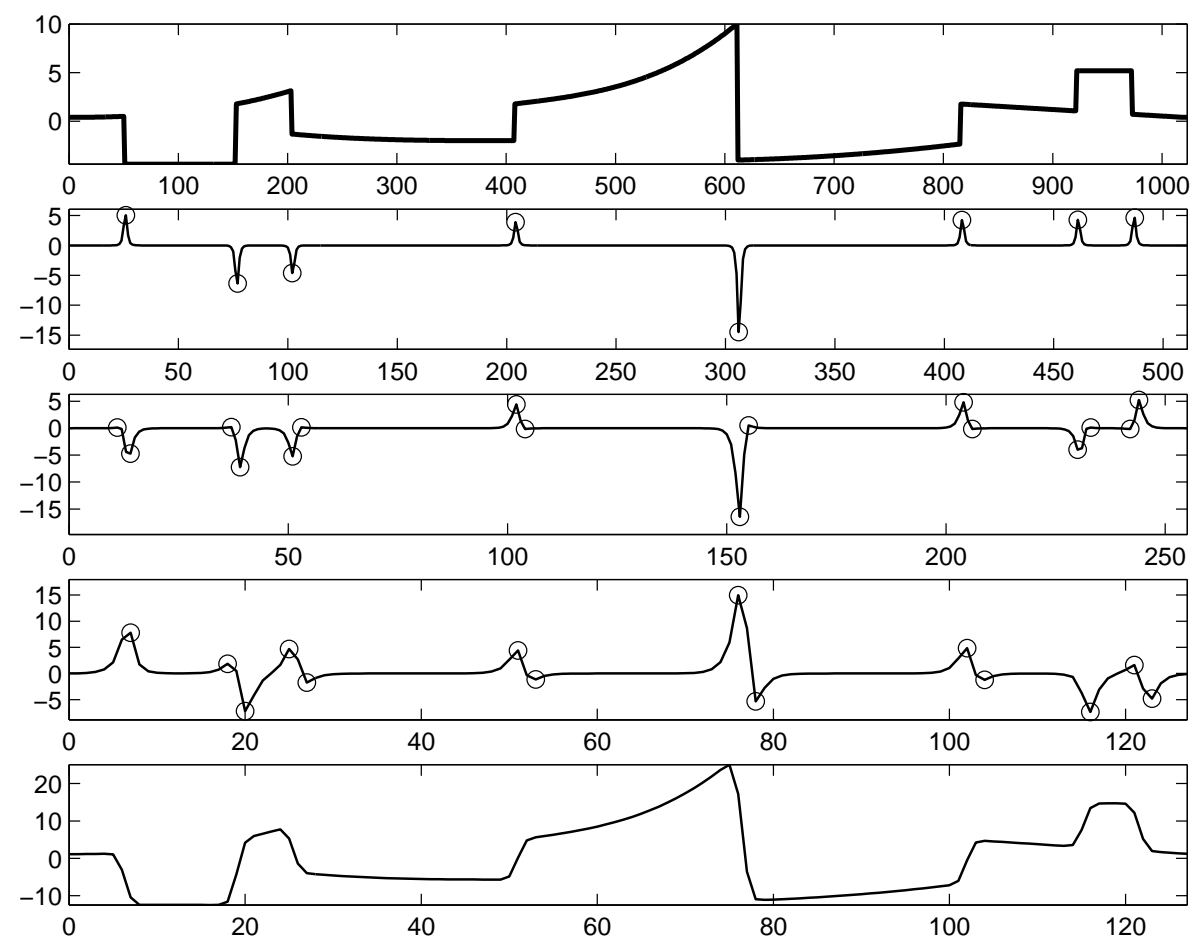

Figure 5. Discrete wavelet transform of a piecewise polynomial signal for $J=3$ iterations, order $\gamma=5$. The local extrema in the subbands are indicated by circles. Differential wavelet at the analysis side.

\section{REFERENCES}

1. S. Mallat, "A theory for multiresolution signal decomposition: The wavelet decomposition," IEEE Trans. Pattern Anal. Mach. Intell. 11, pp. 674-693, 1989.

2. I. Daubechies, "The wavelet transform, time-frequency localization and signal analysis," IEEE Transactions on Information Theory 36(5), pp. 961-1005, 1990.

3. Y. Meyer, Ondelettes et opérateurs I: Ondelettes, Hermann, Paris, France, 1990.

4. S. Mallat, "Zero-crossings of a wavelet transform," IEEE Transactions on Information Theory $\mathbf{3 7}$, pp. 10191033, July 1991.

5. S. Mallat and S. Zhong, "Characterization of signals from multiscale edges," IEEE Transactions on Pattern Analysis and Machine Intelligence 14, pp. 710-732, July 1992.

6. S. Mallat and W. L. Hwang, "Singularity detection and processing with wavelets," IEEE Transactions on Information Theory 38, pp. 617-643, 1992.

7. M. Unser and T. Blu, "Fractional splines and wavelets," SIAM Review 42, pp. 43-67, 2000.

8. T. Blu and M. Unser, "A complete family of scaling functions: The $(\alpha, \tau)$-fractional splines," in Proceedings of the Twenty-Eighth IEEE International Conference on Acoustics, Speech, and Signal Processing (ICASSP'03), IEEE, (Hong Kong), Apr. 2003.

9. C. de Boor, R. Devore, and A. Ron, "Approximation from shift invariant subspaces of $L^{2}\left(\mathbb{R}^{d}\right)$," Trans. Amer. Math. Soc. (341), pp. 787-806, 1994.

10. T. Blu and M. Unser, "Approximation error for quasi-interpolators and (multi-)wavelet expansions," Applied and Computational Harmonic Analysis 6, pp. 219-251, Mar. 1999.

11. M. Unser, A. Aldroubi, and M. Eden, "A family of polynomial spline wavelet transforms," Signal Processing 30, pp. 141-162, 1993.

12. T. Blu, D. Van De Ville, B. Forster, and M. Unser, "Wavelet bases, multiscale differentiation, and solitons," In preparation, 2005. 
13. C. K. Chui and J.-Z. Wang, "A cardinal spline approach to wavelets," Proceedings of the American Mathematical Society 113, pp. 785-793, Nov. 1991.

14. M. Unser, A. Aldroubi, and M. Eden, "On the asymptotic convergence of B-spline wavelets to Gabor functions," IEEE Transactions on Information Theory 38, pp. 864-872, Mar. 1992.

15. T. Blu and M. Unser, "The fractional spline wavelet transform: Definition and implementation," in Proceedings of the Twenty-Fifth IEEE International Conference on Acoustics, Speech, and Signal Processing (ICASSP'00), I, pp. 512-515, (Istanbul, Turkey), June 5-9, 2000.

16. D. Van De Ville, T. Blu, and M. Unser, "Isotropic polyharmonic B-splines: Scaling functions and wavelets," IEEE Transactions on Image Processing, in press. 